\title{
Embedded based smart home automation system
}

\author{
Saurabh Korde ${ }^{1}$, Akshay Waghmare ${ }^{2}$, Abhijeet Inamdar ${ }^{3}$, Akshay Jadhav ${ }^{4}$ \\ Student, Computer Dept, PVPIT, Pune, India ${ }^{1,2,3,4}$
}

\begin{abstract}
In recent 12 month's number of community enabled digital devices are gift at homes. With the quick growth of the net the proprietors were asking for remote controlling on in-home gadgets. This android based domestic automation device lets in a couple of users to govern the software the use of android utility or internet site. The system has 3 issue the ones are mobile device to manipulate the home appliances from any area, $2 \mathrm{~d}$ is server positioned on the cloud which shops statistics associated with home equipment and user and 0.33 device is Wi-Fi router located at home which definitely controls home equipment by sending notification to micro controller.
\end{abstract}

Keywords: Wi-Fi device, cloud, android device, web server, GCM (Google Cloud Messenger).

\section{INTRODUCTION}

The automation is the advent of generation for any infrastructure to decorate running of the gadgets positioned in that infrastructure. Recently the automation turns into a famous field of research by way of the advent of various technologies like remote manipulate structures, network enabled gadgets and net technology. Automation objectives the orchestration of virtual devices to provide consumer real consolation with security and capacity to display a couple of gadgets from any location and at any time. Conventional automation structures contain the controlling of digital devices those provide the capabilities inclusive of heating, lighting and shading.

The blessings of automation structures are indexed as protection, comfort, saving of strength and higher conversation. Because the structures provide these advantages, a few technical requirements additionally required like low cost, plug and play, flexibility, easiness of use and reliability. The machine is designed to serve multiple customers, the use of emerging technologies like $\mathrm{gcm}$ (Google cloud messaging)to assist the verbal exchange among the principle hardware components of the device and cell tool.

\section{RELATED WORK}

There have been significant systems and numerous approaches for the home automation systems. There are several existing automation systems like:

\section{Bluetooth based home automation system:}

This system involves a primary controller and a collection of Bluetooth sub-controllers in which every controller is physically attached to a separate domestic gadgets. The sub-controllers are responsible to switch all messages to number one controller. Although the gadget decreases physical wiring through the usage of the Bluetooth era this generation has the drawback of get right of entry to put off because of the sharing of a single Bluetooth module among numerous devices.
1. Java based automation system:

On this system embedded board which consists into a personal pc based totally internet server is bodily attached to all home devices. Java era used inside the machine offers a built in safety. But, using a high end computer and the stressed out connections in line with home increases the fee of the device.

2. Phone Based System:

An exciting device, phone based faraway manipulate system, is proposed in. in contrast to the severe systems the usage of the internet, the verbal exchange inside the device is all executed over a set phone line. The gain of the machine is that it could be accessed through any smartphone. Then again, the lack of graphical consumer interface, the need to remember the person access code and the device codes may be indexed because the disadvantages.

\section{PROPOSED METHODOLOGY AND DISCUSSION}

Main components of the proposed system are pointed out with diagrams showing the communication infrastructures where those parts are in-use which are as shown below.

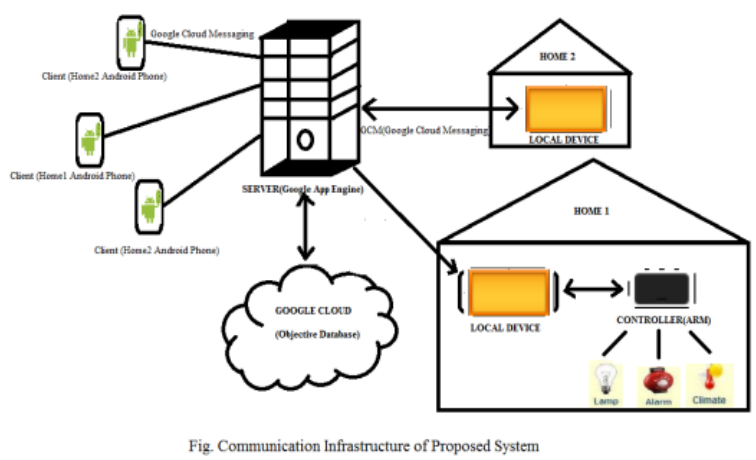

The system involves the following three components: local hardware, web server, and mobile smart device. 
Vol. 5, Issue 12, December 2016

1. Local Hardware:

The nearby hardware includes the $\mathrm{Wi}-\mathrm{Fi}$ tool and the network devices related tohousehold home equipment. After putting in place the nearby hardware and
equipment's at domestic, the proprietor of the proposed home automation gadget enters the home identity given via the administrator of the device and password on to the login web page of the utility in nearby device. On the $\mathrm{gcm}$ server aspect, one-time registration is also necessary. A registration identity is asked from $\mathrm{gcm}$ server and this identification is recorded to the session manager of the application in neighbourhood tool. The web server aspect will apprehend the local tool with that id.

\section{Web Server:}

The net server maintains the person information and serves to the opposite gadgets in the machine. The net server pc gives Google cloud messaging $(\mathrm{gcm})$ service to attach the gadget with the nearby gadgets and the cell devices. Gcm(google cloud messaging) is used for assisting the 2 route verbal exchange between neighbourhood tool and net server and additionally mobile device and internet server. The $\mathrm{gcm}$ carrier handles queuing of messages and delivery to the goal mobile tool. The net server additionally presents an internet website online for the customers of the machine (the clients and the directors) to deal with the statistics saved in the database of the device.

\section{Mobile Device:}

The closing issue is the cell clever tool jogging android running machine like smart phones or tablets, on which the android software of the system is established to make the cell clients contact with and control the in domestic gadgets through the server. the mobile tool application virtually receives the message from the user, stores the message inside the consultation supervisor of the software and transfers statistics to the web server the use of http approach

\section{Modules:}

1. Local Hardware Configuration

2. ARM Controller Configuration

3. Web Server Configuration with GCM service.

4. Android Mobile App for use

5. Web Application Interface.

\section{Mathematical model:}

Set of Input $=\{\mathrm{U}, \mathrm{S}, \mathrm{D}, \mathrm{G}\}$

Set of Output $=\{\mathrm{Msg}\}$

Server $=\{$ IP, MAC $\}$

\section{Where}

G is a GCM (Google Cloud Messaging Services)

Msg is response message

$\mathrm{U}$ is a set of users

$\mathrm{u} 1, \mathrm{u} 2, \mathrm{u} 3$.......un $€ \mathrm{U}$
There can be number of users in our system which interacts with our developed system and uses features of our system.

$\mathrm{S}$ is the Web Server

$\mathrm{D}$ is set of Devices

$d 1, d 2, \ldots \ldots . d n € D$

IP is an IP address of Server ( $\mathrm{S}$ )

MAC is MAC address of Server (S)

\section{CONCLUSION}

The paper proposes a smart automation system using Google Cloud Messaging server and Android operating system as the upcoming technologies used in automation area. The system has three hardware components: a local device to send signals to home appliances, a web server to store customer data and provide services to the other components, and a mobile device running Android application.

\section{ACKNOWLEDGEMENT}

It is indeed a matter of great privilege to publish this paper on "Embedded based smart home automation system" under the valuable guidance of Prof. Mrs S.C. Choudhary. We would like to express our deep sense of gratitude to our guide for this valuable guidance, advice and constant work

\section{REFERENCES}

[1] K. Bromley, M. Perry, and G. Webb, "Trends in Smart Home Systems,Connectivity and Services", www.nextwave.org.uk, 2003.

[2] M. Kovatsch, M. Weiss, and D. Guinard, "Embedding interne ttechnology for home automation", Proc. of ETFA, 2010, pp. 1-8.

[3] F. Moraes, A. Amory, N. Calazans, E. Bezerra, and J. Petrini, "Using theCAN protocol and reconfigurable computing technology for Web-based smart house automation", 14th Symposium on Integrated Circuits andSystems Design, pp. 38-43, 2001.

[4] K. Gill, S.-H. Yang, F. Yao, and X. Lu, "A zigbee-based homeautomation system", IEEE Transactions on Consumer Electronics, vol.55, no. 2, pp. 422-430, May 2009.

[5] N. Sriskanthan, F. Tan, and A. Karande, "Bluetooth based home automation system", Microprocessors and Microsystems, vol. 26, no. 6, pp. 281-289, 2002.

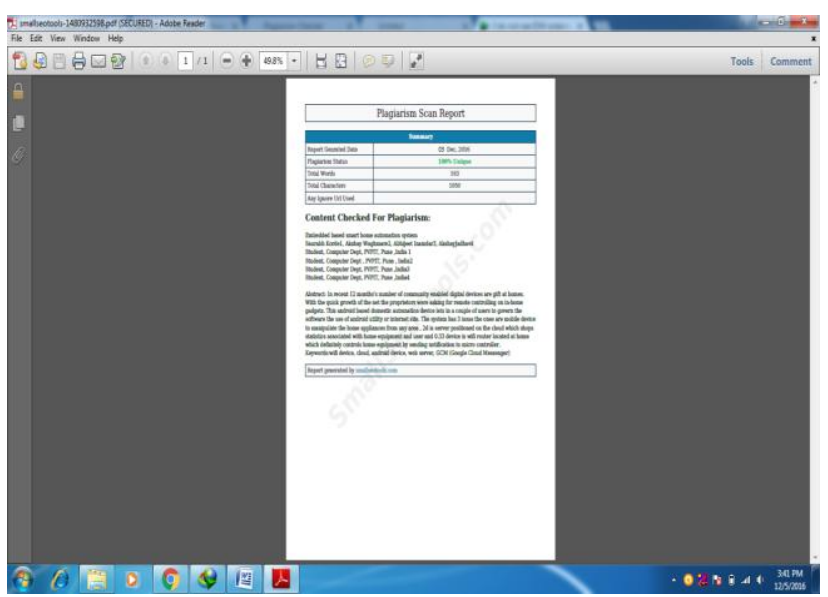

